\title{
Optical design of dual-elliptical mirrors of near-infrared absorption spectroscopy for diabetes detection
}

\author{
Ting-Jou Ding, ${ }^{a}$ Shih-Hsin Ma, ${ }^{b}, *$ and Ker-Ching Chang ${ }^{\mathrm{b}}$ \\ ${ }^{a}$ Ming Dao University, Department of Materials and Energy Engineering, Pitou, Taiwan \\ ${ }^{\mathrm{b}}$ Feng Chia University, Department of Photonics, Taichung City, Taiwan
}

\begin{abstract}
An optical design model of dual-elliptical mirrors near-infrared absorption spectroscopy (NIRAS) for detection of diabetes is proposed. This design model improves the optical efficiency of the conventional NIRAS system without the use of optical components. We used the Henyey-Greenstein scattering skin model in the simulation to calculate the light scattering phenomena. Based on the virtual skin model, the dual-elliptical mirrors NIRAS system can focus the light-emitting diode (LED) light into deeper depths of the human skin and collect more backscattered light. The dual-elliptical mirrors NIRAS system can enhance the light collection efficiency by 1.6 times that of the conventional NIRAS system and 66 times that of the dualelliptical lens NIRAS system. In terms of assembly tolerance, the normalized light collection efficiency (LCE) of LED and photodiode can be maintained above 0.9 and 0.68 , respectively, at a 0.2 -mm displacement. The LCEs of the $\varphi$ and $\theta$ rotations are at least 0.95 with a 5-deg tilt error of the chips. (1) The Authors. Published by SPIE under a Creative Commons Attribution 4.0 Unported License. Distribution or reproduction of this work in whole or in part requires full attribution of the original publication, including its DOI. [DOI: 10.1117/1.OE.60.9.091504]
\end{abstract}

Keywords: light-emitting diode; dual-elliptical mirrors; near-infrared absorption spectroscopy; skin model; diabetes; blood glucose.

Paper 20210114SS received Feb. 1, 2021; accepted for publication Apr. 1, 2021; published online May 4, 2021.

\section{Introduction}

With the abundance of living supplies, most people have become overnourished, and the lack of regular exercise leads to many chronic diseases. Diabetes is one of the chronic diseases related to many serious complications ${ }^{1}$ and has received significant research attention in recent years. Most patients with diabetes use the invasive method, ${ }^{2-5}$ which requires the use of a needle to draw blood, to monitor their blood glucose concentrations. However, this method may cause great psychological pressure and physical pain and even increase the risk of infection.

Therefore, optical non-invasive devices have been developed recently, ${ }^{6-8}$ such as utilizing polarimetry to measure the aqueous humor of the human eyes ${ }^{9,10}$ and optical coherence tomography ${ }^{11}$ to measure the variation of the optical path difference with varying blood glucose concentrations in the skin tissue. In addition, near-infrared absorption spectroscopy (NIRAS) ${ }^{12,13}$ is used to sense the state of blood glucose concentration through the strong absorption signal of blood glucose in the near-infrared band. The NIRAS system has a compact structure and can easily be applied to wearable devices. It usually uses light-emitting diodes (LEDs) ${ }^{14}$ as a light source that have several advantages, such as long life, small size, excellent reliability, high luminosity, and vivid color.

The conventional NIRAS system contains an NIR LED light source and a photodiode (PD). These components are directly attached on the skin surface, as shown in Fig. 1. The divergent light from the LED enters the skin, and then the PD can receive the backscattered light, which contains the related information of blood glucose concentrations.

Under this structure, the efficiency of light energy is poor, and the acquired signal is too small to distinguish and must need an electric amplifier to recognize. To enhance the signal, an optical

*Address all correspondence to Shih-Hsin Ma, shma@fcu.edu.tw 


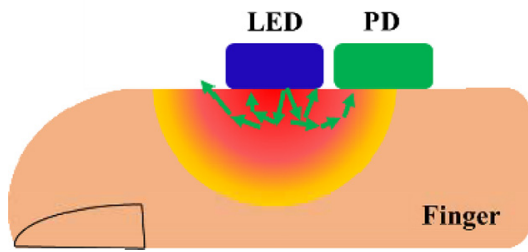

Fig. 1 Schematic of the conventional NIRAS system. The NIR LED light source and PD are directly attached on the skin surface.

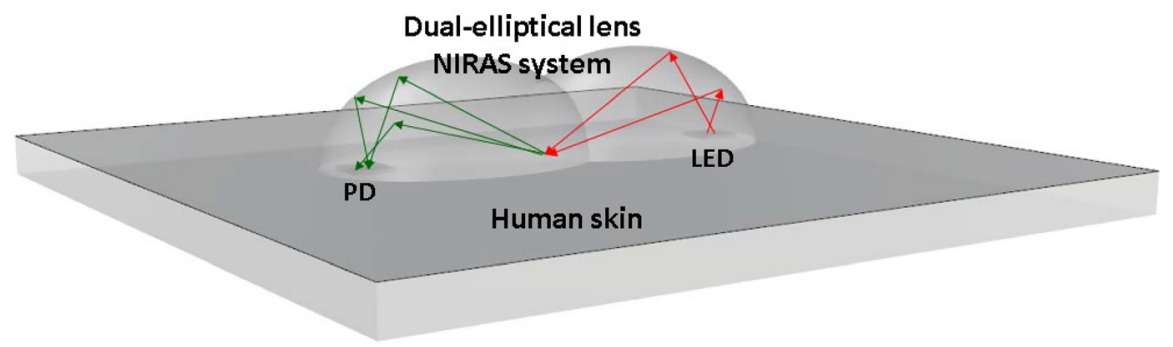

Fig. 2 Structure of the dual-elliptical lens NIRAS system. The dual-elliptical lens can help to focus the emitting light beam and collect backscattering light.

system must be applied to the conventional NIRAS system, as shown in the dual-elliptical lens structure in Fig. $2 .{ }^{15}$

The dual-elliptical lens can help to focus the emitting light from the LED and collect more backscattering light by PD with the total internal reflection (TIR) effect ${ }^{16-18}$ in conventional NIRAS systems. The focus light can increase the penetration depth under the skin, and the other elliptical lens can gather more light to the PD. However, a large amount of light energy is lost from the lens owing to the inferior TIR effect. This system has a flipped LED and a PD laid on the flat side of the elliptical lens and contact with the human skin. In this system structure, chips can dissipate heat and the circuit cannot be easily laid out.

To address these problems, we propose an optical dual-elliptical mirrors NIRAS system, whose mechanism can facilitate heat dissipation because the LED and PD are both away from the skin surface. In the simulation, the assembling tolerance of the LED and PD is taken into consideration.

\section{Optical Design Model of the Human Skin}

The human skin is composed of the epidermis, dermis, and subcutaneous fat layers, as shown in Fig. 3. When light passes through the skin, the skin tissues can be treated as a nonuniform and anisotropic optical medium.

Numerous complex light phenomena, such as scattering, absorption, and interface reflection, occur under the skin. The different characteristics of biological tissues in each layer result in

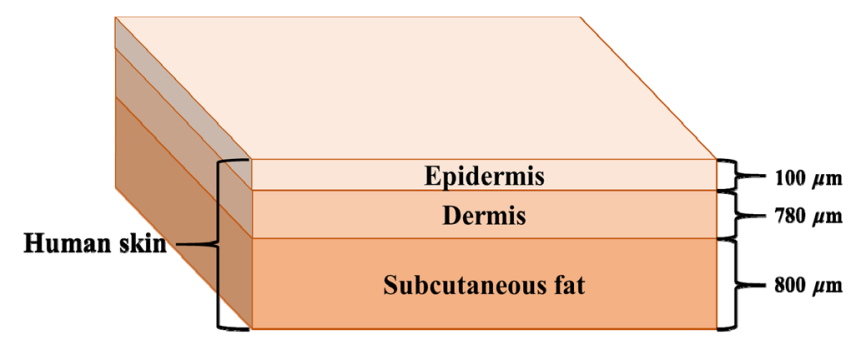

Fig. 3 Side view of the human skin structure, which contains three layers: epidermis, dermis, and subcutaneous fat. 
Ding, Ma, and Chang: Optical design of dual-elliptical mirrors of near-infrared absorption spectroscopy...

Table 1 Optical parameters of the human skin at the 940-nm wavelength.

\begin{tabular}{lccccc}
\hline \hline \multicolumn{5}{c}{ Optical parameters of the human skin } \\
\hline Skin layer & $n$ & $G$ & $\mu_{s}\left(\mathrm{~mm}^{-1}\right)$ & $\mu_{a}\left(\mathrm{~mm}^{-1}\right)$ & Thickness $(\mu \mathrm{m})$ \\
\hline Epidermis & 1.4 & 0.8 & 16.26 & 0.066 & 100 \\
Dermis & 1.4 & 0.8 & 9.77 & 0.08 & 780 \\
Subcutaneous fat & 1.4 & 0.8 & 8.95 & 0.09 & 800 \\
\hline \hline
\end{tabular}

different light phenomena. For this reason, the prediction of scattering light in the skin is very difficult, which affects the light energy efficiency of optical dual-elliptical mirrors NIRAS systems. To analyze the light energy passing through the skin, an optical design model of the skin needs to be built first.

The Henyey-Greenstein scattering model has been widely used in previous research to describe the light scattering behavior in the human skin. ${ }^{19-26}$ The Henyey-Greenstein scattering function is expressed as follows:

$$
p(\theta)=\frac{1}{4 \pi} \frac{1-g^{2}}{\left(1+g^{2}-2 g \cos (\theta)\right)^{\frac{3}{2}}},
$$

where $p(\theta)$ is the probability density function of the scattered light at a specific divergent angle $\theta$ in the biological tissue, $\theta$ is the divergent angle of the scattering light, and $g$ is the anisotropy factor that controls the degree of uniform light scattering, whose value is between -1 and 1 . For instance, a $g$ value of 1,0 , and -1 corresponds to complete forward scattering, isotropic scattering, and complete backscattering, respectively. In this skin model, the Fresnel loss at the interfaces and the light absorption in the medium are dependent on the refractive index $(n)$ and the absorption coefficient $\left(\mu_{a}\right)$ of each layer tissue. In addition, the scattering coefficient $\left(\mu_{s}\right)$ is the probability that the light is scattered after traveling a certain distance in the tissue and is proportional to the concentration of cells in the tissue. We also consider this effect in this study.

To reduce the signal interference caused by the water absorption as much as possible, we chose a 940-nm wavelength LED as the light source. At this wavelength, there is a strong glucose absorption and low water absorption in the human skin. The thicknesses of each layer in this skin model and the corresponding $n, g, \mu_{s}$, and $\mu_{a}$ values at the 940-nm wavelength are listed in Table $1 .{ }^{27}$ The simulation results of a parallel light beam passing through the skin model by the ASAP software are shown in Fig. 4.

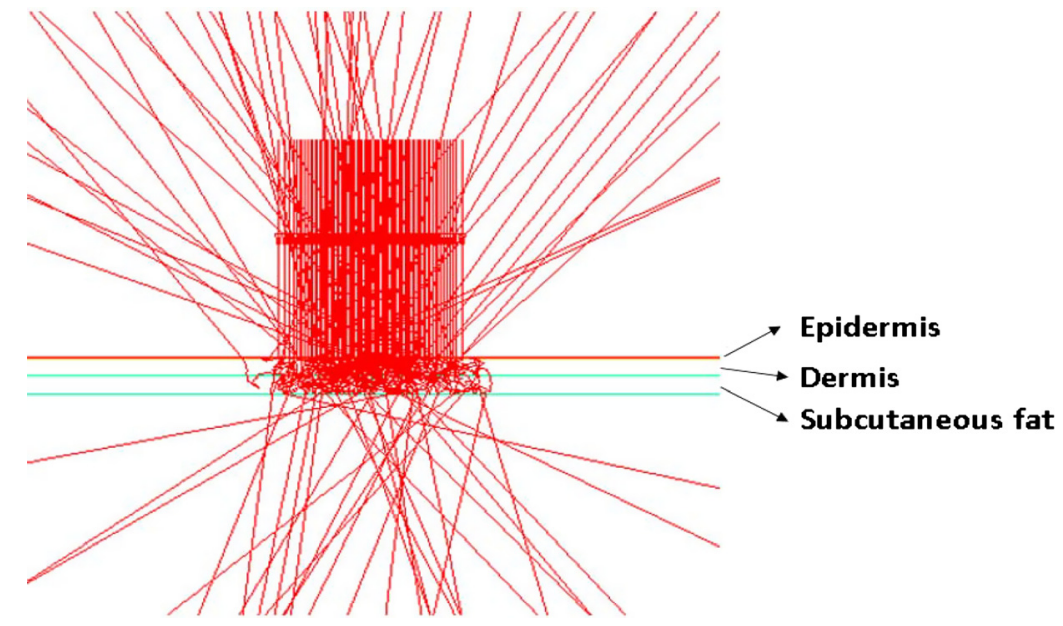

Fig. 4 Simulation results of a parallel light beam passing through the skin model by the ASAP software. 


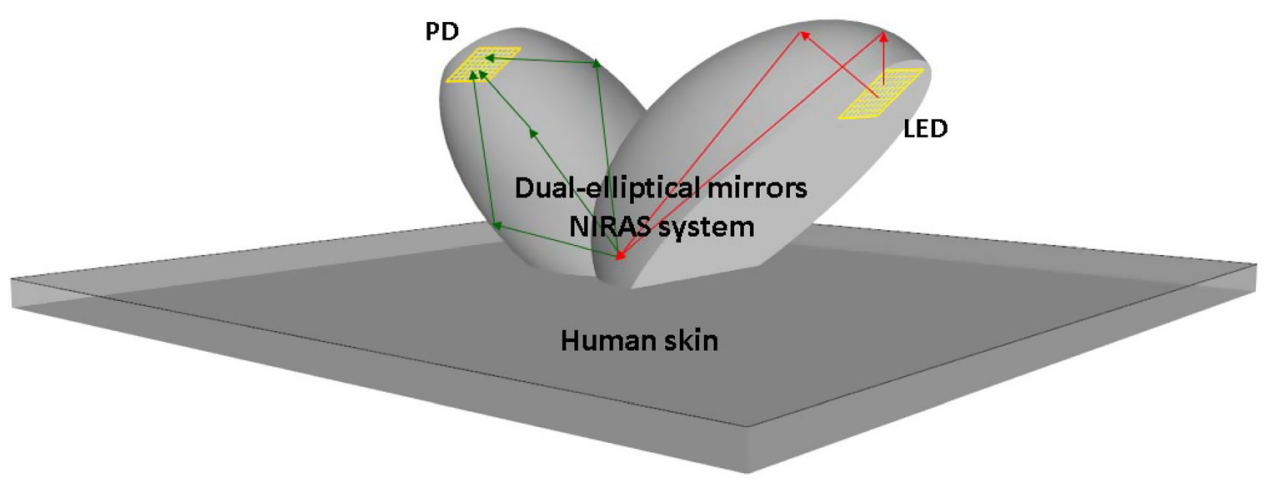

Fig. 5 Structure of a dual-elliptical mirrors NIRAS system.

\section{Light Collection Efficiency Analysis}

In the conventional NIRAS system, an LED is directly attached to the skin surface and near a PD. In such a construction, the light that emerges from an LED is incident into the skin and backscattered immediately, and backscattered light can be detected only in the direction of the PD side. In addition, the size of the PD is too small to collect all the backscattered light in this direction, and most of the light energy scattering from the skin is lost. To obtain more scattering light, a dual-elliptical mirrors NIRAS system composed of two elliptical mirrors is proposed for the improvement of the light collection efficiency (LCE), as shown in Fig. 5.

In our designed system, the LED and PD are both in square shape with a size of $1 \mathrm{~mm} \times 1 \mathrm{~mm}$. In the simulation setup, the LED sight source is set as a Lambertian emitting surface by the Monte Carlo method. The two elliptical mirrors have a confocal point on the surface of the skin. The LED and PD were placed on the second focal points of each elliptical mirror, respectively, and far away from the human skin to avoid thermal damage and to reduce the difficulties of the circuit assembly. The light emitted from the LED can be focused at the location of the confocal point on the skin surface. The focused light beam enters the human skin and increase the light penetration depth. Then, the backscattered light is collected by another elliptical mirror and focused on the PD. The aperture of the dual-elliptical mirrors is located at the confocal point on the skin surface and covers the region where the backscattered light has emerged from the skin. The recess between two elliptical mirrors takes as a function of a shutter that can directly block the light emitted from the LED. We conducted a simulation of three different NIRAS systems, namely, conventional NIRAS systems without optical components, dual-elliptical lens NIRAS system, and dual-elliptical mirrors NIRAS system, through a raytracing method, and the simulation results are shown in Fig. 6.

Some rays are directly incident into the PD through a multi-reflection of an elliptical mirror or the surface of the skin. These rays do not carry the blood glucose information, and all of them are invalid energy that needs to be deducted from an energy calculation. In the simulation, a skin

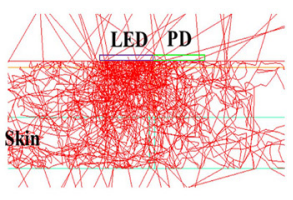

(a)

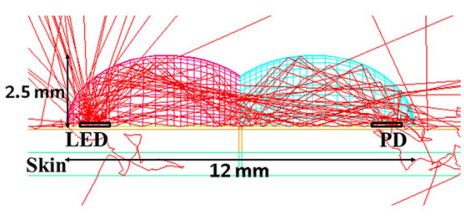

(b)

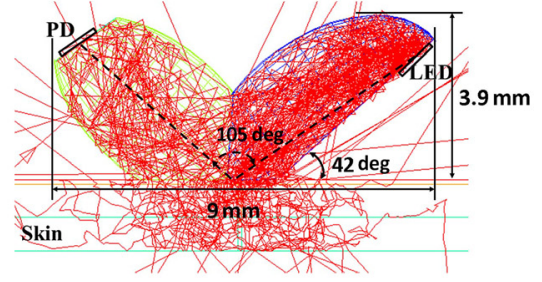

(c)

Fig. 6 Three different NIRAS systems used to measure the blood glucose concentration: (a) conventional NIRAS system without optical components, (b) dual-elliptical lens NIRAS system, its length, width, and height are 12, 4.9, and $2.5 \mathrm{~mm}$, respectively, (c) dual-elliptical mirrors NIRAS system, its length, width, and height are 9,4 , and $3.9 \mathrm{~mm}$, respectively, the angle between the long axes of the two elliptical mirrors is $105 \mathrm{deg}$, and the elevation angle of the ellipse of LED side with respect to the horizontal is $42 \mathrm{deg}$. 


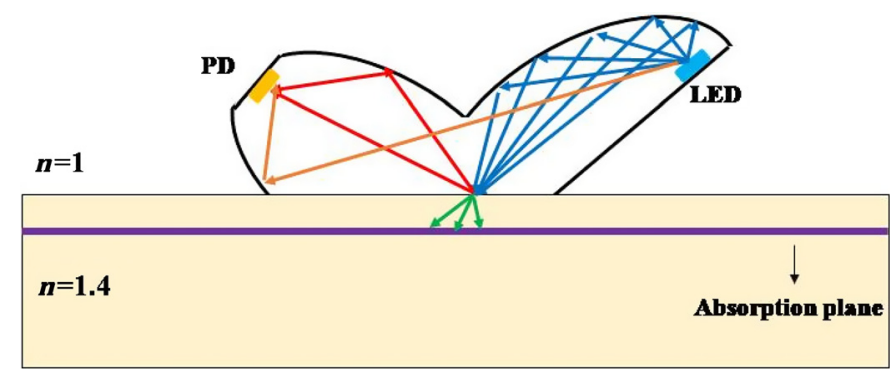

Fig. 7 A skin model without scattering effect is built to calculate the invalid light energy.

model without scattering effect is built to calculate the invalid light energy. The skin model only has a layer of tissue with a refractive index of 1.4. An absorption plane is set in this virtual skin model to absorb the rays in the skin and avoid the rays be reflected back into the air. Then the rays emerged from the LED, and the energy received by the PD is the invalid light energy, as shown in Fig. 7. Hence, the effective light power is expressed as

$$
E_{E}=E_{R}-E_{I},
$$

where $E_{E}$ is the effective light power, $E_{R}$ is the total light power received by the $\mathrm{PD}$, and $E_{I}$ is the invalid light power. The invalid light power includes the light power directly emerging from the LED and the light power reflected by the dual-elliptical mirrors that does not enter the human skin and the power of Fresnel loss of the top skin surface. The ratio of $E_{E}$ and the original light power of the LED is defined as LCE, the ratio of $E_{R}$ and the original light power of the LED is defined as $P_{R}$, and the ratio of $E_{I}$ and the original light power of the LED is defined as $P_{I}$. A comparison of the energy efficiencies of the three is shown in Fig. 8.

In Fig. 9, the dual-elliptical lens NIRAS system has the lowest LCE value of $0.7 \%$. According to the simulation results of the conventional NIRAS system, the PD size, which is just approximately $3 \%$, limits the LCE. However, this structure has hardly any invalid power efficiency of $0.02 \%$ because of the close contact between the chips and skin surface.

In dual-elliptical lens NIRAS systems, the received light power has the largest value, but the invalid power is also larger than that in other systems. The received light power is mostly an invalid power where light does not enter the human skin and carries blood glucose information. Therefore, the LCE in this system is only $0.7 \%$. In the dual-elliptical mirrors NIRAS system, the LCE is $4.8 \%$, which is approximately 1.6 times that of the conventional NIRAS system and 66 times that of the dual-elliptical lens NIRAS system.

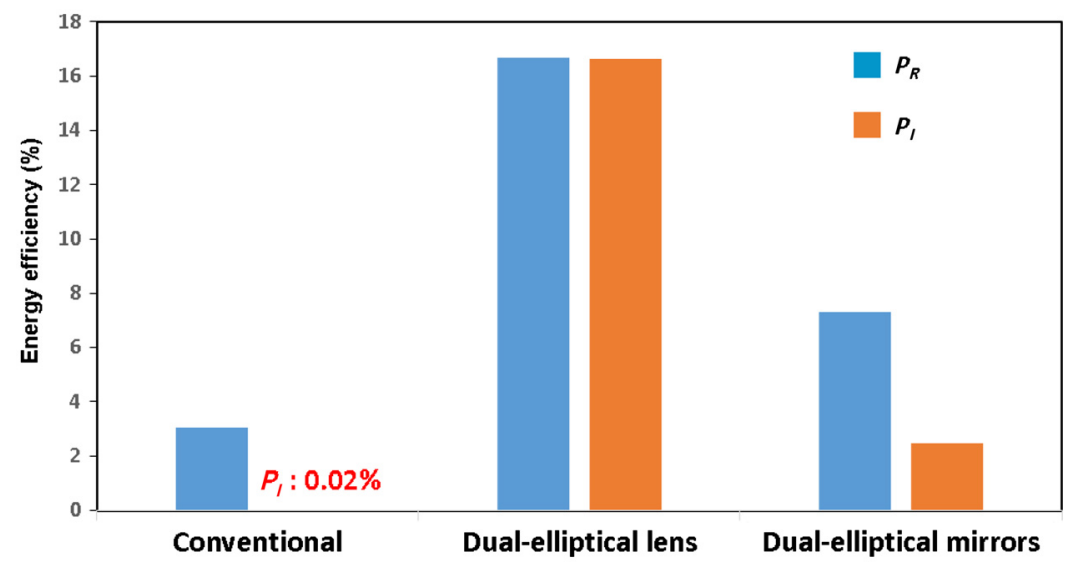

Fig. 8 Comparison of the $P_{R}$ and $P_{1}$ of the conventional, dual-elliptical lens, and dual-elliptical mirrors NIRAS systems. 


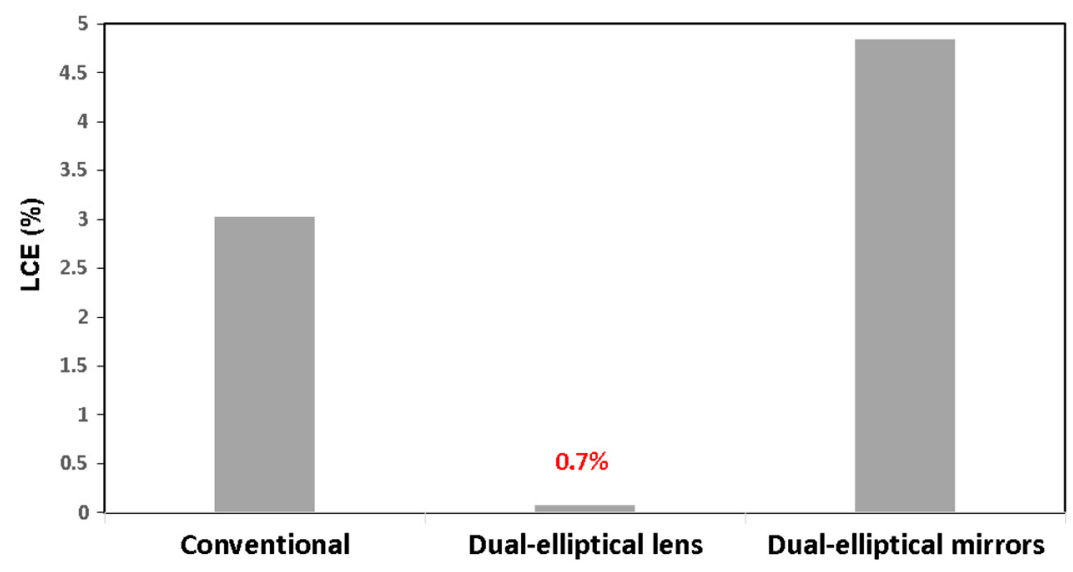

Fig. 9 LCE comparison of the three NIRAS systems.

\section{Assembly Tolerance Analysis}

The assembly errors of the light source, LED, and light receiver, PD, of the optical dual-elliptical mirrors NIRAS system were taken into account, and the tolerance of the efficiency analysis was evaluated by a simulation. Assembly errors, such as position or angle errors, may influence the light collection process. In our simulations, two square holes are designed to assemble LED and PD easily in the dual-elliptical mirrors. The area of the two square holes is both $1 \mathrm{~mm}^{2}$, which is the same as the area of the LED and the PD, as shown in Fig. 10. Figure 11 shows the corresponding coordinate parameters of the LED and PD, where $x$ and $y$ are the coordinate axes, $n$ is the normal of the chips, $\varphi$ is the rotation angle around the $x$-axis, and $\theta$ is the rotation angle around the $y$-axis. The LED and PD were moved around along the normal direction of the chips with a $\pm 0.2 \mathrm{~mm}$ range, as shown in Fig. 11(a). The simulation results are shown in Fig. 11(b), where each displacement of the chips is $0.1 \mathrm{~mm}$. We moved the LED and PD separately and obtained the two curves of LCE. In the normalized LCE trend, the displacements of the chips decreased in the positive and negative normal directions. Based on the analysis results, the decreasing trend caused by moving the LED and PD in the positive normal direction is more severe than that in the negative normal direction. The downward trend includes the defocusing effect caused by the displacement of LED and PD and the loss of some light energy that cannot be reflected by the PD. Furthermore, the influence of the displacement of LED makes it more sensitive than the PD. Whether the LED or PD is utilized, when the moving distance reaches 0.2 and $-0.2 \mathrm{~mm}$, the normalized LCE is still maintained above 0.68 and 0.9 , respectively.

In addition, the angle errors generated during the assembly process are an important issue in the error analysis, and the tilt condition of chips also influences the LCE. In the $\varphi$ direction tolerance analysis, we considered a 5-deg clockwise rotation with a 1-deg interval.

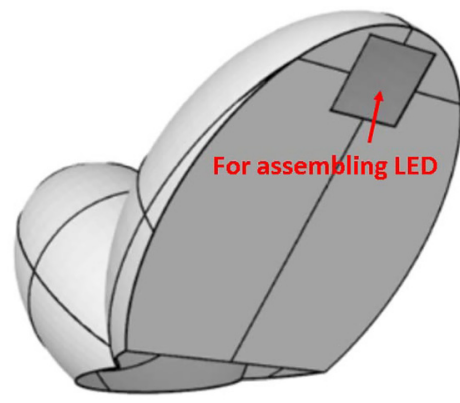

(a)

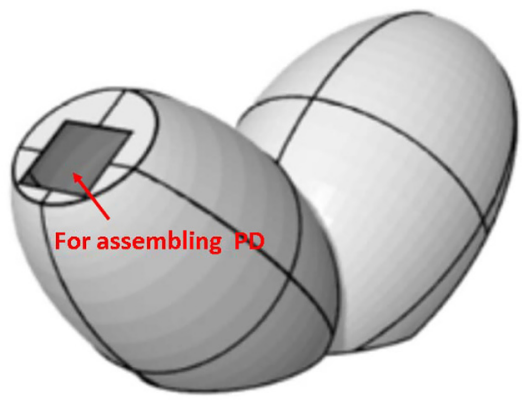

(b)

Fig. 10 Two square holes with 1- $\mathrm{mm}^{2}$ area are designed for easily assembling LED and PD: (a) for assembling LED and (b) for assembling PD. 


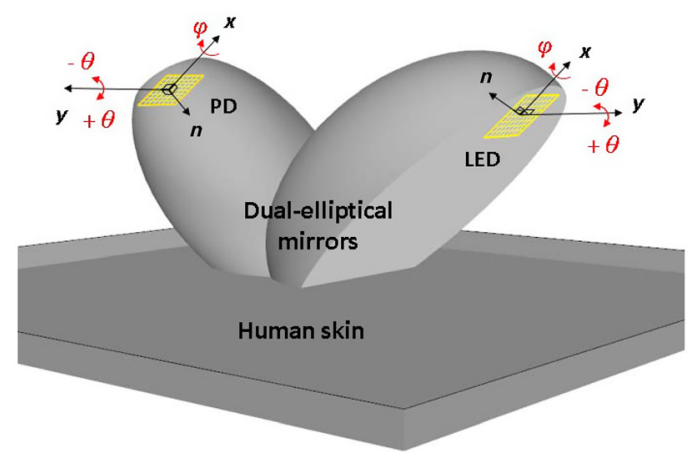

(a)

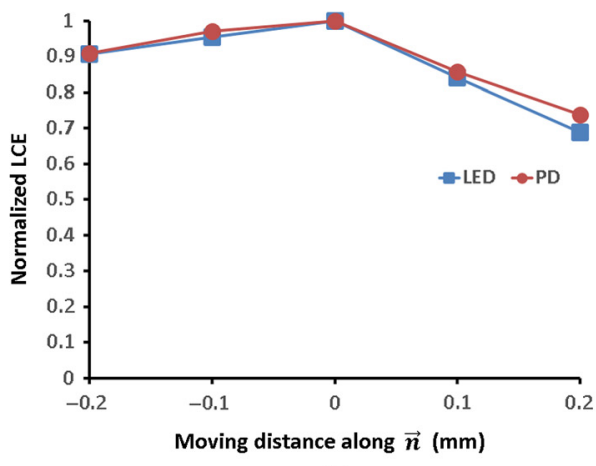

(b)

Fig. 11 Shifting tolerance analysis of the LED and PD: (a) coordinate axis setup of the dualelliptical mirrors NIRAS system and (b) comparison of the normalized LCE with the displacements of LED and PD.

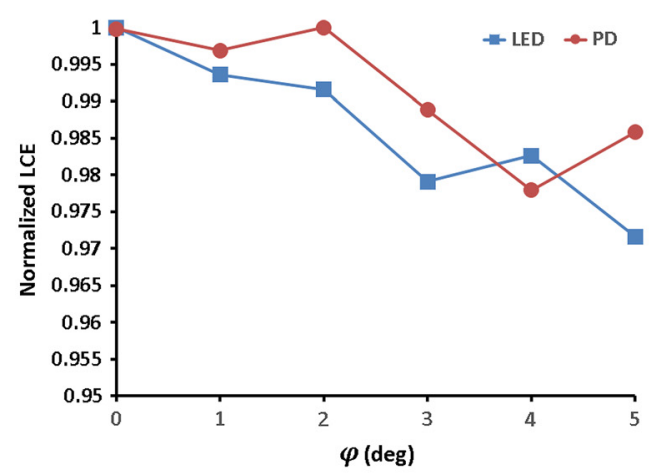

(a)

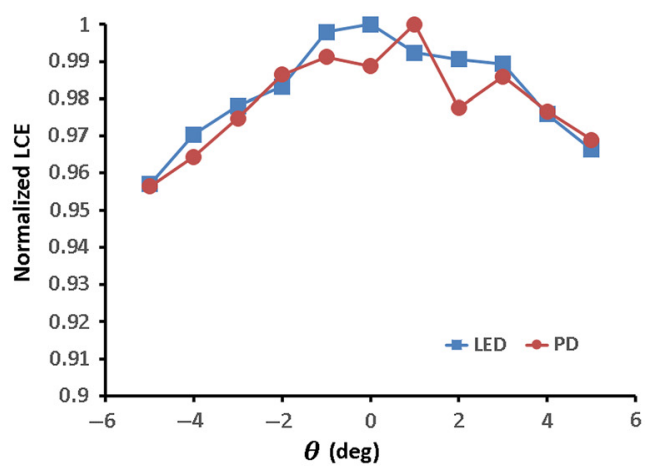

(b)

Fig. 12 Rotating tolerance analysis of the LED and PD. The corresponding normalized LCE in the (a) $\varphi$ direction and (b) $\theta$ direction.

The simulation results are shown in Fig. 12(a). Moreover, we performed a tolerance analysis in the $+\theta$ and $-\theta$ directions. The related normalized LCE is shown in Fig. 12(b).

In the $\varphi$ rotation, the normalized LCEs of the LED and PD dropped slowly. Comparing both of them, the normalized LCE of the LED is slightly more sensitive than that of the PD with the changes in $\varphi$. When the rotation angle reached $5 \mathrm{deg}$, the normalized LCEs were maintained at values above 0.97 . With the changes in $\theta$, a similar trend in the normalized LCEs was observed. Furthermore, the rotation in the $-\theta$ direction is more sensitive than that in the $+\theta$ direction. When the variety of rotation angles reaches $5 \mathrm{deg}$, the normalized LCE can remain above $95 \%$.

Furthermore, the normalized LCE of the PD with a 1-deg rotation angle is higher than that without the rotation angle. Thus, the PD originally placed flat on the top surface of the mirror can successfully increase the LCE, but slightly adjusting the angle of the PD may result in a better LCE performance.

\section{Conclusions}

In this study, an optical design model of the conventional NIRAS system with a dual-elliptical mirror has been proposed to successfully improve the LCE. Based on the Henyey-Greenstein scattering method, a virtual skin optical design model was used to evaluate the LCE in simulations. The dual-elliptical mirrors NIRAS system can effectively focus the light from the LED to deepen the penetration depth in the human skin and collect more backscattered light from the skin. The LCE of the dual-elliptical mirrors NIRAS system is 1.6 times that of the conventional NIRAS system and 66 times that of the dual-elliptical lens NIRAS system. 
Ding, Ma, and Chang: Optical design of dual-elliptical mirrors of near-infrared absorption spectroscopy...

Moreover, in the assembly tolerance analysis, we performed displacement and rotation factor analyses in the simulation and evaluated the effects. According to the simulation results, the decreasing trend of LCE in the positive direction is more severe than that in the opposite direction because of the defocusing effect. The normalized LCEs of the LED and PD can be maintained above 0.68 and 0.9 , respectively, in the 0.2 - and $-0.2-\mathrm{mm}$ displacements. For the chip's rotating analysis, the normalized LCE exhibited a slow downward trend. In the $\varphi$ rotation, the LCE of the LED has slightly more sensitivity than the PD. However, in the $\theta$ rotation, the downtrends of the LCE of the LED and PD are similar. The rotation in the $-\theta$ direction is more sensitive than that in the $+\theta$ direction. Finally, the LCEs of the $\varphi$ and $\theta$ rotations are at least 0.95 with a 5 -deg tilt error in the chips. Thus, in our proposed optical design model, there is a large tolerance for installation errors.

\section{Acknowledgments}

This work was supported by the Ministry of Science and Technology, Taiwan, Project MOST 109-2221-E-035-078.

\section{References}

1. World Health Organization, "Global report on diabetes," 2016, https://www.who.int/ diabetes/global-report/en/.

2. W. X. Xue et al., "Colorimetric detection of glucose and an assay for acetylcholinesterase with amine-terminated polydiacetylene vesicles," Chin. Sci. Bull. 56(18), 1877-1883 (2011).

3. Y. H. Wang et al., "Colorimetric detection of hydrogen peroxide and glucose using the magnetic mesoporous silica nanoparticles," Talanta 134, 712-717 (2015).

4. J. Wang, "Electrochemical glucose biosensors," Chem. Rev. 108(2), 814-825 (2008).

5. H. Yamaoka and K. Sode, "A disposable electrochemical glucose sensor using catalytic subunit of novel thermostable glucose dehydrogenase," Open Biotechnol. J. 1(1), 26-30 (2007).

6. M. J. C. Van Gemert et al., "Skin optics," IEEE Trans. Bio Med. Eng. 36(12), 1146-1154 (1989).

7. W. F. Cheong, S. A. Prahl, and A. J. Welch, "A review of the optical properties of biological tissues," IEEE J. Quantum Electron. 26(12), 2166-2185 (1990).

8. M. K. Chowdhury et al., "Challenges and countermeasures in optical noninvasive blood glucose detection,” Int. J. Innov. Res. Sci. Eng. Technol. 2, 329-334 (2013).

9. C. Chou et al., "Noninvasive glucose monitoring in vivo with an optical heterodyne polarimeter," Appl. Opt. 37(16), 3553-3557 (1998).

10. B. H. Malik and G. L. Coté, "Characterizing dual wavelength polarimetry through the eye for monitoring glucose," Biomed. Opt. Express 1(5), 1247-1258 (2010).

11. S. Amrane, N. Azami, and Y. Elboulqe, "Optimized algorithm of dermis detection for glucose blood monitoring based on optical coherence tomography," in IEEE 2015 10th Int. Conf Intel. Syst.: Theory and Appl., pp. 1-5 (2015).

12. J. Yadav et al., "Near-infrared LED based non-invasive blood glucose sensor," in IEEE 2014 Int. Conf. Signal Proc. and Integr. Networks, pp. 591-594 (2014).

13. K. Maruo et al., "New methodology to obtain a calibration model for noninvasive nearinfrared blood glucose monitoring," Appl. Spec. 60(4), 441-449 (2006).

14. A. Zukauskas, M. S. Shur, and R. Caska, Introduction to Solid-State Lighting, John Wiley \& Sons, New York (2002).

15. Automotive Research \& Testing Center (ARTC), "A driver's physiological monitoring system based on photodiode sensor with reflective optics element," 2016, https://www.artc.org .tw/chinese/03_service/03_02detail.aspx?pid=3057.

16. M. A. Moiseev and L. L. Doskolovich, "Design of TIR optics generating the prescribed irradiance distribution in the circle region," J. Opt. Soc. Am. 29(9), 1758-1763 (2012).

17. F. Chen et al., "Design method of high-efficient LED headlamp lens," Opt. Express 18(20), 20926-20938 (2010). 
Ding, Ma, and Chang: Optical design of dual-elliptical mirrors of near-infrared absorption spectroscopy...

18. I. Wallhead et al., "Design of an efficient Fresnel-type lens utilizing double total internal reflection for solar energy collection," Opt. Express 20(23), A1005-A1010 (2012).

19. V. Tuchin, Tissue Optics: Light Scattering Methods and Instruments for Medical Diagnosis, 2nd ed., SPIE Press, Bellingham, Washington (2007).

20. Y. Fukui, Y. Ajichi, and E. Okada, "Monte Carlo prediction of near-infrared light propagation in realistic adult and neonatal head models," Appl. Opt. 42(16), 2881-2887 (2003).

21. Z. Wang et al., "Monte Carlo simulation of light propagation in human tissue models," in 3rd Int. Conf. Bioinf. and Biomed. Eng., pp. 1-4 (2009).

22. Y. Du et al., "Optical properties of porcine skin dermis between $900 \mathrm{~nm}$ and $1500 \mathrm{~nm}$," Phys. Med. Biol. 46(1), 167-181 (2001).

23. S. Liakat et al., "Mid-infrared $(\lambda=8.4-9.9 \mu \mathrm{m})$ light scattering from porcine tissue," Appl. Phys. Lett. 101(9), 093705 (2012).

24. L. G. Henyey and J. L. Greenstein, "Diffuse radiation in the galaxy," Astrophys. J. 93, 70-83 (1941).

25. D. Toublanc, "Henyey-Greenstein and Mie phase functions in Monte Carlo radiative transfer computations," Appl. Opt. 35(18), 3270-3274 (1996).

26. O. S. Khalil, "Spectroscopic and clinical aspects of noninvasive glucose measurements," Clin. Chem. 45(2), 165-177 (1999).

27. E. V. Salomatina et al., "Optical properties of normal and cancerous human skin in the visible and near-infrared spectral range," J. Biomed. Opt. 11(6), 064026 (2006).

Ting-Jou Ding received his $\mathrm{PhD}$ from the Department of Optics and Photonics, National Central University, Taiwan, in 2012. He is currently an assistant professor in the Department of Materials and Engineering, Ming Dao University, Taiwan. His research interests primarily involve microfluidic systems, application of LEDs, and biomedical sensors.

Shih-Hsin Ma received his $\mathrm{PhD}$ from the Institute of Optical Sciences, National Central University, Taiwan, in 2007. He is currently an associate professor in the Department of Photonics, Feng Chia University, Taiwan. His research interests primarily involve the fundamental investigation and application of LED, especially in the LED optical design of lighting and biomedical science, and quality assessment of LED lighting.

Ker-Ching Chang received his MS degree from the Department of Photonics, Feng Chia University, Taichung City, Taiwan, in 2019. His research interest primarily involves improvement and analysis of the optical mechanism of LED non-invasive blood glucose-measuring devices. 